\title{
Philosophiques
}

\section{Søren Kierkegaard, Correspondance, trad. par A.-C. Habbard, Paris, Éditions des Syrtes, 2004, 464 pages.}

\section{Dominic Desroches}

Volume 32, numéro 2, automne 2005

URI : https://id.erudit.org/iderudit/011889ar

DOI : https://doi.org/10.7202/011889ar

Aller au sommaire du numéro

Éditeur(s)

Société de philosophie du Québec

ISSN

0316-2923 (imprimé)

1492-1391 (numérique)

Découvrir la revue

Citer ce compte rendu

Desroches, D. (2005). Compte rendu de [Søren Kierkegaard, Correspondance,

trad. par A.-C. Habbard, Paris, Éditions des Syrtes, 2004, 464 pages.]

Philosophiques, 32(2), 486-490. https://doi.org/10.7202/011889ar d'utilisation que vous pouvez consulter en ligne.

https://apropos.erudit.org/fr/usagers/politique-dutilisation/ 
Trouillard, Saffrey, Duméry, Hadot, Festugière, et tant d'autres, évoqués bien sûr dans l'ordre chronologique, à l'exception de quelques retours en arrière à des fins explicatives. Les notices sont assez brèves, WH n'entre que rarement dans le détail des analyses et des textes, et il préfère le plus souvent s'en remettre à des sources intermédiaires dont il donne en quelque sorte de petites fiches de lectures (par exemple, les considérations sur le spiritualisme reprennent essentiellement les indications des travaux de D. Janicaud), ou bien à des articles ou des livres d'entretiens.

L'histoire des idées est ici bien à la peine. WH cultive davantage l'anecdote que l'analyse, et il se plaît à souligner que "Armstrong était lui-même décidément anticlérical» (p. 201), que J.-L. Marion, historien de la philosophie, « est aussi un leader parmi les théologiens catholiques» (p. 224), que L. Brisson "naquit dans un petit village agricole du Québec» (Platon; 243) et que, grâce à J.-M. Narbonne, un «renouveau de la philosophie » est à prévoir (p. 257-258). Voilà qui est édifiant, sans doute, mais l'examen des arguments philosophiques est en reste. On apprend certes que "chez Bréhier, comme chez Bergson, l'arrière-plan idéaliste est présent " (p. 144), mais guère plus.

La question que posait WH conserve son intérêt. Il y a bien une actualité des études néoplatoniciennes, et cette actualité contemporaine tient pour beaucoup à une quête spirituelle liée à l'Église. Mais répondre à cette question eût supposé, par exemple et très simplement, que l'on se souciât de ce que des lecteurs chrétiens ou christianisants vont chercher et trouver dans le texte plotinien, de la manière dont ils accordent ces textes à leur quête, en négligent ou en corrigent certains aspects ou arguments. Rien de tel ici. Cette lacune est curieusement exacerbée par la publication conjointe des deux essais : réfléchissant sur le premier et sur la manière dont le souci de "l'au-delà "s'y manifeste si vivement, le second aurait pu y trouver matière à réflexion.

JEAN-FRANÇOIS PRADEAU

Université de Paris $\mathrm{X}$ - Nanterre et Institut Universitaire de France

\section{Søren Kierkegaard, Correspondance, trad. par A.-C. Habbard, Paris, Éditions des Syrtes, 2004, 464 pages.}

Voici une excellente nouvelle pour le monde de la recherche francophone sur Kierkegaard: la compilation des lettres et des billets de Kierkegaard, les Breve og Aktstykker vedrørende Søren Kierkegaard (Munksgård, 1953-54), vient d'être traduite en langue française. Cela constitue en quelque sorte un soulagement, car le lecteur francophone, qui ne disposait pas de ce matériel unique et précieux (ces documents ne font pas partie des œuvres complètes), n'était pas en mesure de saisir les enjeux «souterrains» de la production kierkegaardienne. Avant de se pencher sur le contenu de la correspondance, nous nous proposons de rappeler le contexte dans lequel paraît ce document si important pour la compréhension de l'œuvre de Kierkegaard en général et pour la recherche sur certains thèmes en particulier.

Tout d'abord, cette traduction vient compléter le travail entrepris il y a plusieurs années dans le Nachlaß et les lettres par les premiers traducteurs de Kierkegaard. On se souviendra ici que Paul-Henri Tisseau avait proposé une première traduction de Lettres à Régine Olsen (Bazoges-en-Pareds) en 1949. Sous le titre Lettres de fiançailles 
(Falaize), Marguerite Grimault, surtout intéressée par la psychologie kierkegaardienne et le drame des épousailles, avait réuni, en 1956, quelques lettres de Kierkegaard à sa fiancée Régine, tout en ajoutant les lettres envoyées à son ami Emil Boesen, afin de restituer le contexte dans lequel s'était produit la célèbre rupture. Si ce livre permettait au lecteur de s'imaginer comment Kierkegaard jouait au fin stratège à Berlin (il utilisait en effet son ami Boesen comme informateur en lui donnant des consignes précises pour espionner Régine...) et à quel point il estimait «sa " petite Régine, cela ne disait toutefois rien du rapport que le penseur de Copenhague entretenait avec les membres de sa famille et les personnalités de l'époque. Or, en 1998, une nouvelle étape dans le travail de traduction de la correspondance est franchie lorsque AnneChristine Habbard, ancienne élève de l'École normale supérieure, fait paraître dans Philosophie quelques «Lettres choisies sur l'amour et le don». Son but est alors de montrer que Kierkegaard est plus que le penseur de la mélancolie, de l'angoisse et du désespoir puisqu'il se penche avec bonheur, dans certaines de ses lettres, sur l'amour, le don et la grâce, ce qui fait aussi de lui un grand philosophe de l'éthique. En si bon chemin, Habbard a eu la force de proposer, et il faut l'en remercier deux fois plutôt qu'une, la traduction complète des lettres de Kierkegaard, ce qu'il convient de voir ici.

Or, que trouvera-t-on dans ce document clé pour la recherche? Après l'intéressante introduction (11-50), la traductrice Habbard propose une liste des correspondants. Ces quelques pages, auxquelles le lecteur est appelé à revenir en cours de lecture, auront le mérite de présenter synthétiquement toutes les personnes à qui Kierkegaard s'est adressé au moyen de l'écrit. Sur le modèle de classification proposé dans les Breve og Atkstykker par N. Thulstrup, la correspondance est ensuite divisée en trois périodes: la période de jeunesse (1829-1841) durant laquelle Søren étudie à Copenhague et s'éprend de la petite Régine Olsen, la période de maturité (1842-1847) durant laquelle il publie l'essentiel de ses ouvrages philosophiques (pseudonymes) et théologiques (édifiants) et, enfin, la période plus polémique, à la fin de sa courte vie, durant laquelle il critique viscéralement l'Église d'État danoise, tout en répondant, dans l'Instant, aux attaques et caricatures dont il est victime dans les journaux (18471855). À ces lettres, la traductrice a ajouté celles dont on ne peut déterminer la date ou le destinataire, un utile arbre généalogique de la famille Kierkegaard (460) ainsi qu'une chronologie des œuvres (461-462). Cela dit, passons sans tarder au contenu des Breve.

Les premières lettres (1829-1841) montrent un Kierkegaard cherchant à mieux se comprendre lui-même. Durant cette période, fait à noter, si le jeune Søren correspond cordialement avec son frère aîné Peter Christian, il ne recevra toutefois qu'une seule lettre de son père, Michael Petersen, pour qui il jouait, avant de déménager, le rôle de secrétaire. Il n'y aura qu'une seule «lettre du père ", dont le contenu importe peu sur le plan philosophique [lettre 4]. Alors qu'il est inscrit comme étudiant en théologie à l'université, Kierkegaard, qui reçoit dès 1836 une missive du critique Johann Ludwig Heiberg parce qu'il lui a envoyé son premier livre, Des papiers d'un homme encore en vie, entreprend une correspondance décisive avec son ami Emil. Celle-ci durera jusqu'en 1850. Après le décès de son père en 1838, il se lie avec Régina Olsen. La plupart des lettres adressées à Régine, qui ne sont pas datées (!), illustrent tout le travail de distance qui habite Kierkegaard et qui résume sa pensée éthique. En effet, le jeune écrivain, qui s'amuse à espacer les mots d'entête (À ma petite Régine), écrit dans une lettre [7]: «Espacer» signifie éloigner les mots les uns des autres; et 
donc, quand j'espace les mots ci-dessus, je cherche à les éloigner à une distance telle qu'un typographe en perdrait certainement patience, car il en viendrait alors probablement à ne rien composer d'autre dans sa vie» (95). À distance, Kierkegaard sait cependant se rapprocher de Régine, tout en restant secret. La correspondance, et c'est décisif, témoignera magnifiquement des jeux de distance qui orientent la pensée de Søren. Dans une lettre très célèbre écrite durant cette période par exemple [17], Kierkegaard se dessine lui-même traversant le pont de Knippels pour aller rejoindre sa fiancée... Mais si pour lui la relation à Régine relève de l'éternité, il précise [lettre 27]: "la distance entre nous s'évanouit, et tu es mienne, unie à moi, un continent dût-il nous séparer» (108). Cette distance se traduira aussi par des mises en scène stratégiques: en grand romantique, l'amoureux Kierkegaard passe sous la fenêtre de Régine au moment même où le coursier met la lettre dans la boîte aux lettres (113). Ici, la correspondance nous donne une idée de la place importante de la communication existentielle dans la vie de l'auteur.

La période suivante (1841-1847) - et la correspondance le montrera avec précision - renforce l'idée d'un Kierkegaard attentif au moindre détail concernant sa vie privée. En effet, s'il part de nouveau pour Berlin en 1841, donc après la rupture avec Régine, il n'a pas oublié sa fiancée pour autant. Les lettres envoyées à Emil [49 et 50], déjà publiées en français, trahissent, on l'a dit, le caractère contrôlant de l'étudiant. Kierkegaard, qui lui donne ses ordres, se montre surpris lorsqu'Émil ne fait pas exactement ce qu'il lui demande, lorsqu'il ne suit pas son "plan » à la lettre. Pourtant, à Berlin, Kierkegaard doit poursuivre sa formation en philosophie en assistant aux cours de Marheinecke et Schelling. Søren, qui "travaille très fort », ira jusqu'à donner des comptes rendus des exposés de Schelling à son frère, à Emil et à Henriette Lund, comme si ces derniers pouvaient saisir ses critiques subtiles... [54]. Ces lettres, toutefois, sont très utiles pour saisir la manière dont l'étudiant danois Kierkegaard interprète la pensée allemande de l'auteur du Système de l'idéalisme transcendantal. Durant cette période berlinoise, $\mathrm{Ou}$ bien... Ou bien... est en préparation et exige beaucoup de ses forces, car il fait froid en cet hiver de 1842.

Mais la correspondance de cette période témoignera aussi des soucis financiers du jeune écrivain. En effet, Kierkegaard, qui a hérité de son père, apprend que le cours de ses actions est en baisse à la bourse de Copenhague [68]. Or, s'il doit rentrer dans la capitale après ses études, ce n'est pas pour devenir plus riche, mais pour devenir écrivain. Cela explique en partie que, dès son arrivée à Copenhague, sa pensée «retourne» en Allemagne! En 1843, Kierkegaard reprendra le vapeur pour Berlin. Et, comme on le sait, ce sera durant ce second séjour, que, encore amoureux de Régine, il rédigera son discours édifiant sur Job ainsi que La répétition et Crainte et Tremblement. Les lettres à son espion danois (Emil) demeurent nombreuses, comme celles adressées aux membres de la famille Lund, Henriette, Carl et Michael. On relèvera ici - un détail peut-être - que Kierkegaard cherche, malgré la distance, à respecter soigneusement (éthiquement?) les dates d'anniversaire, surtout celle d'Henriette, tout se passant exactement comme s'il n'avait pas le droit de les oublier. Si Kierkegaard, dès son retour, tente d'apprivoiser sa récente célébrité, il suit de près la publication de ses derniers livres et il continue sa correspondance avec Heiberg, Sibbern et le pasteur Spang. On pourra lira ici non sans intérêt les billets à Israel Levin, son secrétaire. Les lettres de cette seconde période, somme toute, illustreront un véritable souci d'éthique et montreront également combien Kierkegaard savait utiliser sa correspondance pour publiciser son œuvre d'écrivain. 
La dernière période (1847-1855) sera, à plusieurs titres, la plus riche en rebondissements puisque le Magister veille à la diffusion de ses idées. La correspondance servira en quelque sorte de correctif aux incompréhensions qu'engendre son œuvre. Si l'auteur se retrouve au centre de polémiques, il écrit plus de lettres. On verra comment Kierkegaard, devenu un maître pour sa génération, fait circuler stratégiquement ses livres dans Copenhague. Ce n'est donc pas un hasard si, en 1847, Kierkegaard retravaille ses lettres aux éditeurs Philipsen et Reitzel [153 à 157]. Mais la correspondance prend aussi un tour éthico-religieux lorsque l'épistolier, qui refuse de guider des lectrices passionnées, laisse voir des signes de vulnérabilité. On trouvera en effet des traces de cette vulnérabilité dans les lettres qu'il projette d'envoyer à Régine (il lui en expédiera une) et dans celle qu'il adresse à Schlegel, l'époux de celle-ci, en 1849 [235 à 239], lettres dans lesquelles il revient sur son passé. S'il fait des promenades et correspond régulièrement avec le professeur Kolderup-Rosenvige et Rasmus Nielsen, en qui il voit un mauvais disciple, il n'est plus en mesure de supporter son frère aîné, Peter Christian, devenu "nuisible» au moment même où celui-ci est en bute avec l'Église. Heureusement pour nous, Peter Christian n'a pas brûlé, contrairement aux voeux de Søren, les lettres entre les deux frères. La dernière lettre conservée [300], datée de juillet 1855, est pour l'éditeur Reitzel: Kierkegaard donne son accord pour la publication du numéro 2 de l'Instant, son feuillet personnel contre l'Église danoise.

Ainsi, la lecture de la correspondance aura réservé quelques surprises. À la fin de sa vie par exemple, jamais Kierkegaard ne se plaint des attaques dont il souffre de la part du journal Corsaren. Loin de se plaindre, il garde le silence sur ses souffrances. Il n'utilise ses lettres que pour se rapprocher des personnes, c'est-à-dire qu'il se "sert » de sa correspondance pour pratiquer l'éthique, les Papirer servant à élaborer ses idées personnelles et ses projets de livre. Très souvent, il se retrouve dans la position du conseiller (tout particulièrement lorsqu'il écrit aux membres de la famille Lund) et joue ce rôle à merveille, sauf avec Emil évidemment, qu'il instrumentalise sans trop s'en formaliser. Il écrit toujours, en style libre, sur un ton courtois, poli et sympathique. Et s'il a un faible pour les formulations latines, s'il se réfère à la littérature classique et ne refuse jamais l'ironie, il écrit sans crainte au "je », se confie, et sait, par là, tirer profit du mode épistolaire. Kierkegaard n'a jamais joué à l'homme important ou au génie - il aimait visiblement trop les autres pour cela -, voilà ce que dévoile magnifiquement sa correspondance inédite.

Nous devons saluer ici la persévérance de Anne-Christine Habbard, de même que son équipe, pour ce travail de grande qualité. Car les chercheurs francophones pourront enfin étudier Kierkegaard comme correspondant, ce qui permettra de révéler de nouvelles facettes de l'homme. Si Kierkegaard est déjà connu comme un "philosophe de journaux ", il ne l'est pas encore pour sa correspondance, contrairement à Platon, Kant, Nietzsche ou d'autres. Avec ce document unique, peut-être verrons-nous en Søren Kierkegaard un correspondant d'un genre tout particulier. Cela ne saurait tarder, car si les Papirer ne sont que partiellement traduits en français, les lecteurs devront lire cette Correspondance pour comprendre la conception que le Magister se faisait du voyage, de sa famille, de son unique ami, de l'écriture, de son œuvre, voire du rapport à autrui. Notons un dernier point: si Kierkegaard ne donne pas dans ses lettres la clef ou le secret de son œuvre d'écrivain, il se montre cependant sous un jour nouveau, précisément comme un homme rigoureux et ouvert, critique et tolérant, sérieux et ironique, paradoxes bien « kierkegaardiens » qui contrasteront agréablement 
avec les préjugés les plus solides à l'endroit du philosophe danois. Ce document précieux, parce qu'il assure une meilleure intelligence de la genèse et du développement de la pensée de Kierkegaard, constitue un outil de référence indispensable pour toute personne qui s'intéresse à la biographie, à l'écriture et à la pensée de Kierkegaard. Nous en recommandons donc vivement la lecture.

DOMINIC DESROCHES

Université de Montréal 